\title{
Interstitial pneumonitis-like lesions in guinea-pigs following repeated exposure to toluene dilisocyanate
}

\author{
K. Yamada+*, R. Amitani+, A. Niimi+, F. Kuze+
}

Interstitial pneumonitis-like lesions in guinea-pigs following repeated exposure to toluene diisocyanate. K. Yamada, R. Amitani, A. Niimi, F. Kuze. CERS Journals Ltd 1995.

ABSTRACT: The inhalation of isocyanates, such as toluene diisocyanate (TDI), diphenylmethane diisocyanate (MDI), and hexamethylene diisocyanate (HDI) can induce hypersensitivity pneumonitis (HP) as well as bronchial asthma in humans, but the precise pathological features and their pathogenetic mechanisms have not been elucidated. To provide insight into the pathological features of isocyanateinduced hypersensitivity pneumonitis in humans, we repeatedly exposed guinea-pigs to TDI following previous sensitization to TDI and examined the inflammatory response in the pulmonary lesions.

Following sensitization with $10 \%$ TDI ethyl acetate solution for seven consecutive days, guinea-pigs were exposed to $5 \%$ TDI ethyl acetate solution once a week for 4 weeks. As a control, guinea-pigs were exposed to ethyl acetate alone in the same manner. Furthermore, other guinea-pigs received a single exposure to 5 or $20 \%$ TDI ethyl acetate solution. The TDI solutions or ethyl acetate were applied to the bilateral nasal mucosa of guinea-pigs for $30 \mathrm{~s} \cdot \mathrm{day}^{-1}$.

Histological examination of lung specimens of guinea-pigs repeatedly exposed to TDI after previous sensitization by TDI inhalation revealed interstitial pneumonitis-like lesions in which mononuclear cells and eosinophils were mainly involved. Lungs of control and nonsensitized guinea-pigs showed insignificant histological changes.

We demonstrated that interstitial pneumonitis-like lesions, indistinguishable from isocyanate-induced hypersensitivity pneumonitis in humans, can be caused by repeated but not single exposure to TDI in guinea-pigs.

Eur Respir J.,1995, 8, 1300-1306.
+Dept of Infection and Inflammation, Chest Disease Research Institute, Kyoto University, Kyoto, Japan. *First Department of Internal Medicine, Osaka Medical College, Osaka, Japan.

Correspondence: K. Yamada First Dept of Internal Medicine Osaka Medical College

Daigaku-Cho 2-7

Takatsuki-City

Osaka

Japan

Keywords: Eosinophils guinea-pig

interstitial pneumonitis repeated exposure toluene diisocyanate

Received: May 311994 Accepted after revision April 101995
The inhalation of isocyanates, such as toluene diisocyanate (TDI), diphenylmethane diisocyanate (MDI), and hexamethylene diisocyanate (HDI), has been known to induce several types of respiratory diseases in humans [1]. TDI is the most widely-used of the isocyanates that can cause bronchial asthma [1-3] and pulmonary lesions which are reported as hypersensitivity pneumonitis (HP) [4-11], alveolar disruption [12], or haemorrhagic pneumonia [13] in humans.

Since the first report on HP induced by TDI or HDI by CHARLES et al. [4], there have been several reports on isocyanate-induced HP in humans. Diagnosis of isocyanate-induced HP was obtained in most cases either by clinical symptoms, abnormal chest radiograph and restrictive ventilatory impairment [4], by positive specific antibodies to isocyanate-human serum albumin [69], or by a positive provocation response to isocyanates [5, 7-11]. There have been few reports $[4,11]$ on the pathological features of isocyanate-induced HP, and the pathogenetic mechanisms of HP induced by TDI are not yet as well understood as organic dust-induced HP.

As it is often difficult to perform open lung biopsy in acute lung diseases, including isocyanate-induced HP, an animal model is required to elucidate human isocyanateinduced HP. To our knowledge, suitable animal models of isocyanate-induced HP have not been developed. Although CHARLES et al. [4] developed a rabbit model of interstitial pneumonitis associated with TDI, the animals were sensitized by intradermal injection of TDI-egg albumin, not by inhalation through the airways.

To determine whether interstitial pneumonitis similar to isocyanate-induced HP is caused by repeated airborne exposure to TDI in guinea-pigs, and to observe the kinds of inflammatory cells involved, we exposed guinea-pigs repeatedly to TDI. The airways exposure was performed after transnasal sensitization to examine the pathological changes of the lungs provoked in a condition similar to that which may cause TDI-induced HP in humans.

\section{Material and methods}

\section{Animals and chemicals}

Male Hartley guinea-pigs, weighing 250-300g on arrival, were purchased from Shizuoka Laboratory Animal Center 
(Shizuoka, Japan). The animals were maintained in clean conditions in the Laboratory of Animal Experiments for Infection and Immunity, in Chest Disease Research Institute, Kyoto University. The animals were carefully examined for 1 week prior to use in the experiments, and only healthy animals were used in the study.

The TDI used 2,4-TDI purchased from Nakalai Tesque (Kyoto, Japan).

\section{Exposure to TDI}

TDI ethyl acetate solutions (5\% and $10 \%)$ were prepared immediately before use. Since ethyl acetate is a volatile organic solvent and does not react with TDI, TDI can be inhaled into the airways and lungs [14]. To induce pulmonary lesions, animals were exposed to TDI by the following method: the TDI solution $(10 \mu \mathrm{L})$ was applied to the bilateral nasal mucosa of guinea-pigs for $30 \mathrm{~s}$ with a thin cotton applicator. Control guinea-pigs received only ethyl acetate with the same technique.

\section{Experimental design}

Eighty seven guinea-pigs were divided into five groups (Group I: n=7; Group II: n=55; Group III: n=5; Group IV: $n=10$; Group V: $n=10$ ) according to mode of exposure. Furthermore, Group II was divided into six subgroups (A-E: $n=10 ; F: n=5)$. Groups IV and V were divided into two subgroups each $(A: n=5 ; B: n=5)$. Group I was exposed only to ethyl acetate as a control. Group II was exposed to $10 \%$ TDI solution once daily for seven consecutive days for sensitization. The animals in the other groups (Groups III, IV and V) were not sensitized. During the repeated exposures to 10 and 5\% TDI solutions for 5 weeks, six animals in Group II died; five of the six animals that died showed deep laboured abdominal breathing. Characteristics of the groups are summarized in table 1.

Group I $(n=7)$. Ten microlitres of ethyl acetate was applied to the nasal mucosa for seven consecutive days, followed 7 days later by repeated exposures to ethyl acetate once a week for 4 weeks. The animals were killed $6 \mathrm{~h}$ after the last exposure.

Group II $(n=49)$. Ten microlitres of $10 \%$ TDI solution was applied to the nasal mucosa for seven consecutive days, followed 7 days later by repeated exposures to $5 \%$ TDI solution once a week for 4 weeks. The animals were killed: 30 min (subgroup A: $n=7$ ); 2 h (subgroup B: $n=9$ ); 3 h (subgroup C: $n=10$ ); 6 h (subgroup D: $n=8$ ); 24 h (subgroup E: $n=10$ ); or 7 days (subgroup $F: n=5$ ) after the last exposure, respectively.

Group III (n=5). Ten microlitres of 5\% TDI solution was applied to the nasal mucosa once a week for 4 weeks. The animals were killed $6 \mathrm{~h}$ after the last exposure.

Group IV $(n=10)$. Ten microlitres of 5\% TDI solution was applied to the nasal mucosa; the animals were killed $2 \mathrm{~h}$ (subgroup A: $n=5$ ), or $6 \mathrm{~h}$ (subgroup B: $n=5$ ) later.

Group V $(n=10)$. Ten microlitres of $20 \%$ TDI solution was applied only once to the nasal mucosa; the animals were killed $2 \mathrm{~h}$ (subgroup A: $\mathrm{n}=5$ ), or $6 \mathrm{hr}$ (subgroup B: $\mathrm{n}=5$ ) later.

After each exposure to TDI solution or ethyl acetate, each animal was checked for wheezing, deep laboured abdominal breathing, and paradoxical respiration which suggested asthmatic reactions.

\section{Histological examination}

Each animal was anaesthetized intraperitoneally with sodium pentobarbital $\left(50 \mathrm{mg} \cdot \mathrm{kg}^{-1}\right)$, and was exsanguinated from an external jugular vein. Additional injections were given as necessary. The trachea was cannulated. The lung was then inflated with $10 \%$ formalin via the tracheal cannula to the pressure of $20 \mathrm{cmH}_{2} \mathrm{O}$ during 10 min. The thoracic cavity was opened to remove the left lung. The lower lobes of the left lungs were sectioned along the left lower lobe bronchus. After preparation in slices, fixation was performed for 5-7 days with $10 \%$ formalin. The sections were then embedded in paraffin,

Table 1. - Characteristics of experimental groups and procedures

\begin{tabular}{cccccc}
\hline $\begin{array}{c}\text { Experimental } \\
\text { group }\end{array}$ & $\begin{array}{c}\text { Guinea-pigs } \\
\mathrm{n}\end{array}$ & $\begin{array}{c}\text { Sensitization } \\
(\text { daily } \times 7 \text { days) }\end{array}$ & $\begin{array}{c}\text { Nasal application } \\
\text { (once a week) }\end{array}$ & $\begin{array}{c}\text { Time-point* after } \\
\text { last exposure }\end{array}$ \\
\hline I & & 7 & Ethyl acetate & Ethyl acetate $(\times 4)$ & $6 \mathrm{~h}$ \\
II & A & 7 & $10 \%$ TDI & $5 \%$ TDI $(\times 4)$ & $30 \mathrm{~min}$ \\
& B & 9 & $10 \%$ TDI & $5 \%$ TDi $(\times 4)$ & $2 \mathrm{~h}$ \\
& C & 10 & $10 \%$ TDI & $5 \%$ TDI $(\times 4)$ & $3 \mathrm{~h}$ \\
& D & 8 & $10 \%$ TDI & $5 \%$ TDI $(\times 4)$ & $6 \mathrm{~h}$ \\
& E & 10 & $10 \%$ TDI & $5 \%$ TDI $(\times 4)$ & $24 \mathrm{~h}$ \\
III & F & 5 & $10 \%$ TDI & $5 \%$ TDI $(\times 4)$ & 7 day \\
IV & A & 5 & - & $5 \%$ TDI $(\times 4)$ & $6 \mathrm{~h}$ \\
& B & 5 & - & $5 \%$ TDI $(\times 1)$ & $2 \mathrm{~h}$ \\
V & A & 5 & - & $5 \%$ TDI $(\times 1)$ & $6 \mathrm{~h}$ \\
& B & 5 & - & $20 \%$ TDI $(\times 1)$ & $2 \mathrm{~h}$ \\
& & 5 & - & $20 \%$ TDI $(\times 1)$ & $6 \mathrm{~h}$ \\
\hline
\end{tabular}

\footnotetext{
*: animals in each group were killed at this time-point. TDI: toluene diisocyanate solution.
} 
sliced $4 \mu \mathrm{m}$ in thickness, and were stained with haematoxylin and eosin (H\&E). Two slices were examined histologically for each animal by an observer who was not aware of the nature of the specimens.

All available fields of left lower lobe were viewed directly through a light microscope at magnification $\times 60$ to $\times 200$. Histological changes in the alveolar regions were scored for pathological features based on the criteria by RICHERSON et al. [15] indicated as follows: $0=$ clean; $0.5=$ few nondescript cellular collections; $1.0=$ mild scattered alveolitis; $2.0=$ moderate scattered alveolitis; $3.0=$ severe alveolitis; $4.0=$ severe alveolitis with alveolar filling.

Epithelium of the large bronchus with cartilage from the orifice of the left lower lobe bronchus to immediately above the segmental bronchi was viewed directly through a light microscope at magnification $\times 400$, and six fields were chosen randomly from each animal. The number of inflammatory cells accumulated in the airway mucosa of the left lower lobe bronchus was counted and expressed as the number of cells $\cdot \mathrm{mm}^{-1}$ length of the airway basement membrane, which was measured with an objective micrometer.

\section{Statistical analysis}

All results were expressed as mean \pm SEM. Nonparametric analysis of variance (Kruskal-Wallis method) was used to determine the significance of variance among the 12 groups. If a significant variance was found, a MannWhitney U-test was used to analyse the significance of differences between individual groups, and since more than one comparison was made, differences with a $\mathrm{p}$ value of less than 0.01 were considered significant. Data were analysed with a computer using standard statistical packages.

\section{Results}

\section{External symptoms}

In 40-45 animals in Group II, deep laboured abdominal breathing occurred between $30 \mathrm{~min}$ and $6 \mathrm{~h}$ after each nasal application of 5\% TDI solution. Deep laboured breathing was observed in 2-4 out of 5 animals in Group III after the third and fourth nasal application of $5 \%$ TDI solution. By contrast, no deep laboured abdominal breathing was observed in the control group (Group I) or the single exposure groups (Groups IV and V).

\section{Pathological features of pulmonary lesions}

Most animals in the control group (Group I) and all unsensitized groups (Groups III, IV and V) showed almost normal alveoli containing a few dispersed macrophages (fig. 1).

Cellular infiltrations in the alveolar septa were present in the four sensitized subgroups (IIB, C, D and E) that had been killed 2-24 h, respectively, after the last exposure. In the animals graded 2.0, alveolitis was characterized by infiltration of mononuclear cells and eosinophils into the alveolar septa (figs $2 \mathrm{~A}$ and B). Lymphocytes comprised the majority of the infiltrating cells, with fewer histiocytes and eosinophils. Few neutrophils were found, and fibrosis was negligible. Infiltration of inflammatory cells extended into the alveolar ducts and involved some of the respiratory bronchioles. Small nonnecrotizing granulomas were also found. These granulomas contained a mixture of epithelioid histiocytes, multinucleated giant cells, lymphocytes and eosinophils and tended to be loosely formed and poorly circumscribed. Vasculitis was not found in any specimen. The characteristic pathological findings described above seem to be similar to those of isocyanate-induced HP in humans $[4,11]$. In the animals graded 4.0, the reaction was similar in character but more severe, and alveolitis was accompanied by alveolar filling (fig. 3A). Eosinophils were prominent among cells infiltrating into the alveolar space (fig. 3B).

\section{Pathological grading of pulmonary lesions}

Repeated exposure to TDI following sensitization by TDI inhalation caused pulmonary lesions in the guinea-pigs. The pathological changes varied considerably between animals within the same group. However, the pathological gradings of the animals in the three sensitized subgroups (IIB, C and E), that had been killed 2, 3 and $24 \mathrm{~h}$, respectively, after the last exposure, were significantly higher $(\mathrm{p}<0.01)$ than those in the control group (Group I) or those in the unsensitized groups (Groups III, IV and V). The most marked pathological changes were observed in the animals in the sensitized Group IIE that had been killed $24 \mathrm{~h}$ after the last exposure. Histological examination of the sensitized Group IID that had been killed $6 \mathrm{~h}$ after the last exposure also revealed pathological changes which were not significantly different to those in the control group or the unsensitized groups. The pathological grading of two sensitized subgroups (IIA and F), that had been killed $30 \mathrm{~min}$ and 7 days, respectively, after the last exposure, was similar to that in the control group or the unsensitized groups (fig. 4).

\section{Airway morphological features}

In the mucosa of the left lower lobe bronchus of each animal in Groups II and III, the accumulation of eosinophils was prominent (fig. 5). Airway leucocytes other than eosinophils were either absent or present in extremely small numbers in all groups.

The numbers of eosinophils accumulated in the mucosa of the left lower lobe bronchus of the animals in Groups II and III that had been repeatedly exposed to 5\% TDI solution were significantly larger $(\mathrm{p}<0.01)$ than those in the control group (Group I) or the single exposure groups (Groups IV and V) (fig. 6). 


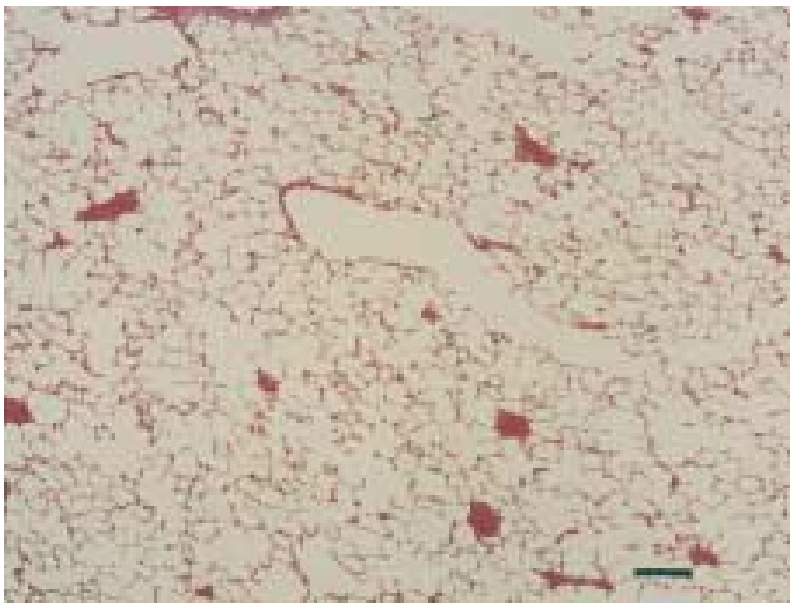

Fig. 1. - Control guinea-pig lung (from Group I), Normal lung of ethyl acetate exposed control guinea-pig receiving once a week challenge with ethyl acetate for 4 weeks (haematoxylin and eosin staining; internal scale bar=30 $\mu \mathrm{m}$ ).
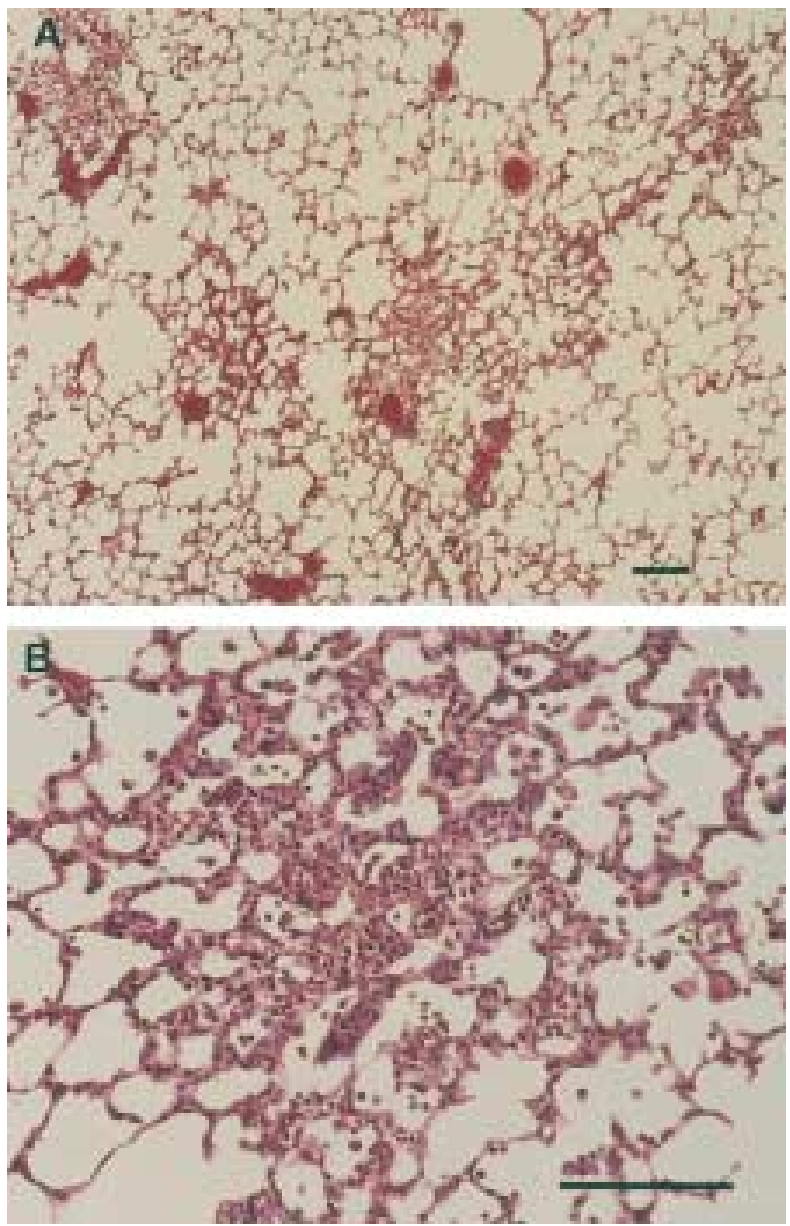

Fig. 2. - Grade 2.0 reaction in the lung of toluene diisocyanate (TDI) exposed guinea-pig that received once a week challenge with TDI for 4 weeks and was killed $24 \mathrm{~h}$ after the last challenge (from Group IIE). A) Scattered areas of alveolitis of moderate severity. B) Same animal, higher magnification showing alveolar wall thickening with lymphocytes and eosinophils (haematoxylin and eosin staining; internal scale bar: $30 \mu \mathrm{m})$
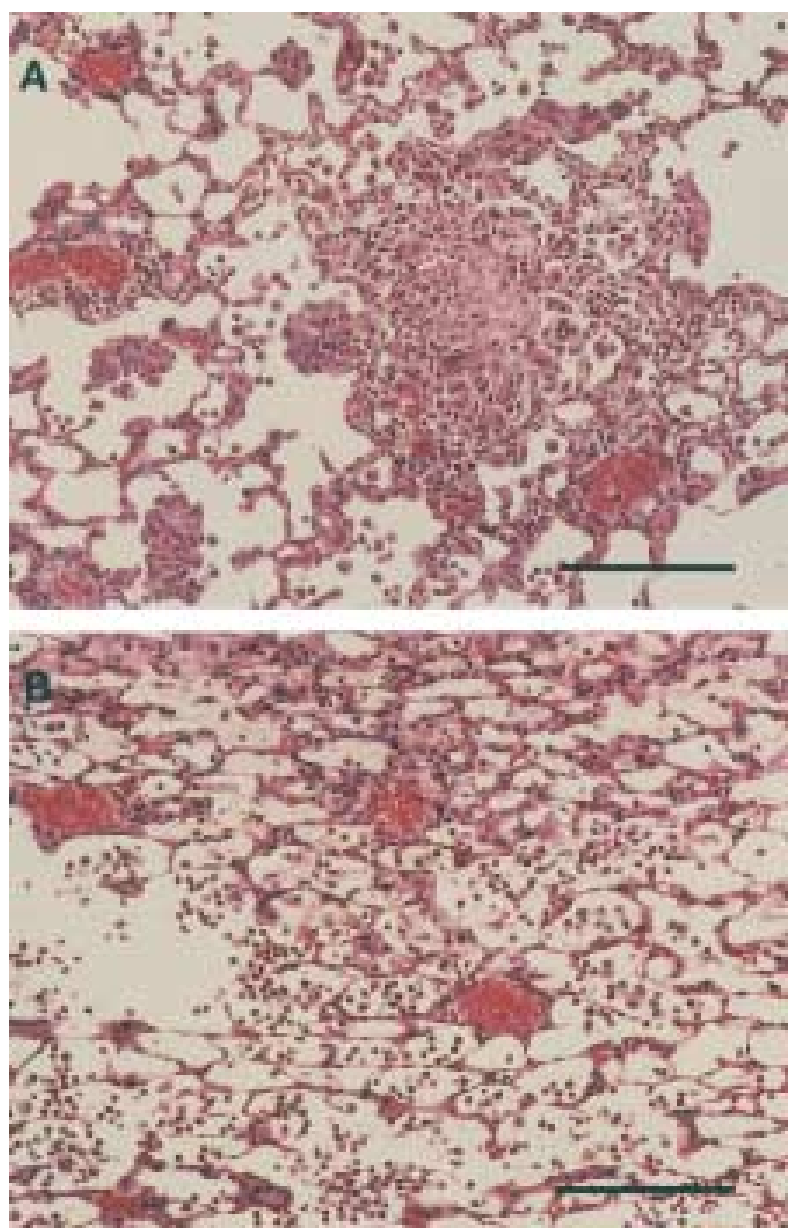

Fig. 3 - A) Grade 4.0 reaction in lung of toluene diisocyanate (TDI) exposed guinea-pig that received once a week challenge with TDI for 4 weeks and was killed $24 \mathrm{~h}$ after the last challenge (from Group IIE). Extensive areas of alveolitis, showing non-necrotizing granuloma and alveolar wall thickening. B) Grade 4.0 reaction in lung of another Group IIE guinea-pig. Extensive areas of alveolitis with alveolar filling of eosinophils and mononuclear cells (haematoxylin and eosin staining; internal scale bars $30 \mu \mathrm{m}$ ).

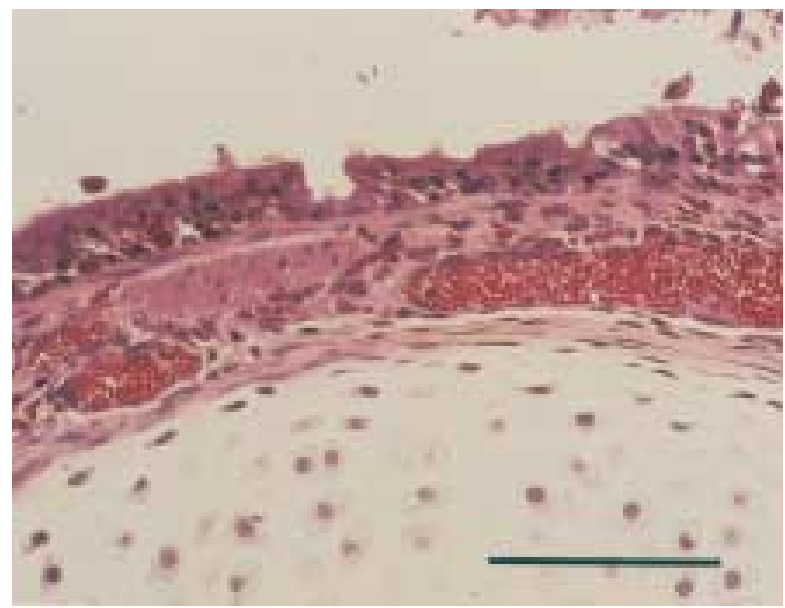

Fig. 5. - Representative photomicrograph of left lower lobe bronchus (from Group IID). Marked eosinophil accumulation in airway mucosa (haematoxylin and eosin staining; internal scale bar $=20 \mu \mathrm{m}$ ). 


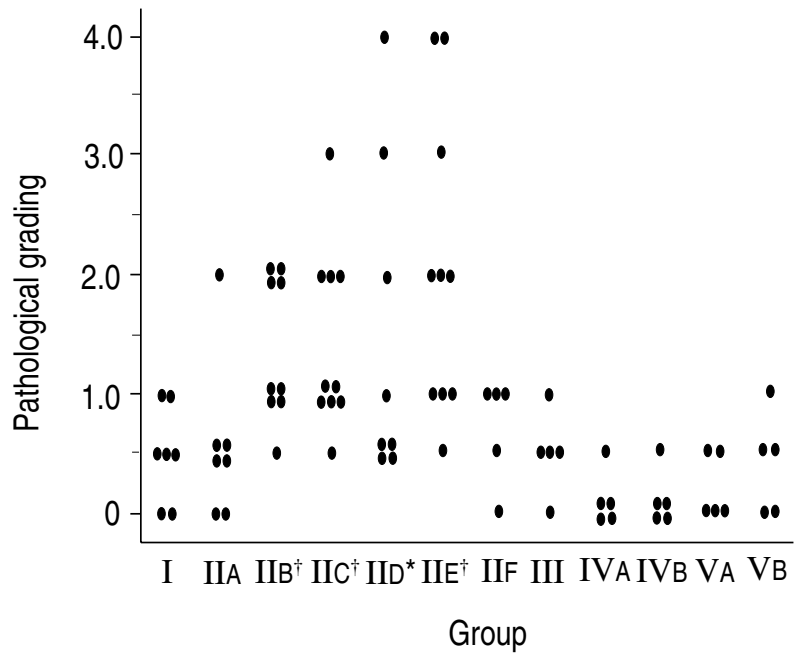

Fig. 4. - Pathological gradings of pulmonary lesions in groups I-V (see table 1 for the individual group treatments). Pathological grading: $0=$ clean; $0.5=$ few nondescript cellular collections; $1.0=$ mild scattered alveolitis; $2.0=$ moderate scattered alveolitis; $3.0=$ severe alveolitis; $4.0=$ severe alveolitis with alveolar filling. The score for each individual guinea-pig is given. $\dagger$ : $p<0.01$ compared with Groups $I$, and III-V; * $: \mathrm{p}<0.05$ compared with Groups I, and III-V.

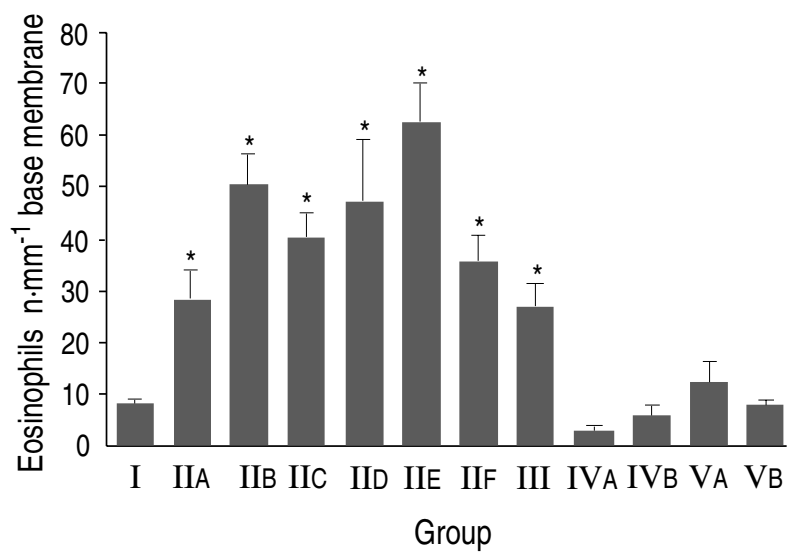

Fig. 6. - Mean numbers ( $\pm \mathrm{SEM})$ of eosinophils $\cdot \mathrm{mm}^{-1}$ length of basement membrane of left lower lobe bronchus Groups I-V (see table 1 for individual group treatments). There is a significant increase in number of eosinophils in groups IIA-F and III compared to that of Groups I, IVA, IVB, VA and VB $(*$ : $\mathrm{p}<0.01)$.

In Groups II and III, marked eosinophil infiltration was observed in the small airways without cartilage as well as in large airways with cartilage.

\section{Discussion}

The present study shows that repeated exposure to TDI after sensitization by nasal application of TDI can cause interstitial pneumonitis-like lesions in guinea-pigs. These lesions are characterized by the following pathological features; 1) infiltration of mononuclear cells into alveolar septa with or without epithelioid granuloma; and 2) an influx of eosinophils into both alveolar septa and alveolar spaces. The abnormalities seen in our animals are similar to these observed in isocyanate-induced HP in humans $[4,11]$.
Although the exposure to isocyanates has been known to induce HP as well as bronchial asthma in humans [1], the pathological features and pathogenetic mechanisms of isocyanate-induced HP in humans have not been fully elucidated. Only a few studies $[4,11]$ have evaluated the pathological features, which include collagenous thickening with (mononuclear) lymphocytes in the alveolar wall, and heavy infiltration of eosinophilic polymorpho-nuclear cells in the interalveolar septa, and intraalveolar cellular exudation. These findings bear a striking resemblance to the lesions in guinea-pigs reported here. These histological changes are also in line with the findings caused by organic dust particles [16], but are different from the organic dust-induced HP with regard to the influx of eosinophils into the alveolar septa and alveolar spaces demonstrated in the present study.

As it is difficult to study the histological changes of HP induced by repeated exposure to isocyanates in humans in its acute phase, the development of an animal model is required to provide insight into the different phases of isocyanate-induced HP. So far, the reported animal models of pulmonary diseases induced by isocyanates have been focused on bronchial lesions [17, 18]. The experimental animal model of so-called interstitial pneumonitis induced by isocyanates has been reported by Charles et al. [4], who developed the rabbit model of interstitial pneumonitis induced by TDI-egg albumin antigen. In rabbits sensitized to produce precipitating antibodies against TDI-egg albumin and challenged with the same antigen, they reported polymorphonuclear cellular exudation, peribronchial lymphoid hyperplasia, interstitial inflammatory changes, pulmonary oedema, and some vasculitis but no eosinophilic responses. These histological changes did not resemble those in isocyanateinduced HP in humans mentioned above $[4,11]$. CHARLES et al. [4] stated that TDI-egg albumin, used in their study as an antigen, is unlikely to be the same as that produced in the body by the combination of TDI vapour and body proteins. They also postulated that it is more likely that TDI reacts with the protein of the mouth, nose and upper respiratory tract, and that particles of conjugated protein are then inhaled. CHARLEs et al. [4] also stated that further studies focused on the relationship between TDI vapour-body protein complex and lesions in the lungs similar to those obtained with TDI-egg albumin conjugate were required. In addition, in the experiment reported by CHARLEs et al. [4], sensitization was achieved intradermally and the challenge was performed by a single endotracheal instillation of antigen, which were conditions different from those in which the isocyanateinduced HP occurs in humans.

In contrast to the study of CHARLEs et al., [4], we repeatedly exposed guinea-pigs to TDI to mimic the condition of HP caused in humans. The pathological changes in the present study include: 1) infiltration of mononuclear cells into the alveolar septa with or without epithelioid granuloma; and 2) an influx of eosinophils both into alveolar septa and alveolar spaces. These pathological changes are similar to the isocyanate-induced HP in humans. 
We found many eosinophils but not neutrophils, in the airways of guinea-pigs that had been repeatedly exposed to TDI. SAETTA and co-workers [19, 20] showed that, in patients with occupational asthma induced by TDI, the bronchial mucosa exhibited accumulation of eosinophils, mast cells and mononuclear cells, but not neutrophils. The airway morphological changes in the guinea-pigs in the present study resemble the airway inflammation occurring in bronchial asthma induced by TDI. Isocyanateinduced HP often occurs with bronchial asthma [6, 8-11]. The presence of interstitial pneumonitis-like lesions and bronchial lesions similar to isocyanate-induced asthma in the present study suggests that the interstitial pneumonitis-like lesions in our experiment resemble the HP induced by TDI in humans. However, further studies are required to explain the difference between the inflammatory cells involved in the lung parenchyma and the airways. KARR et al. [21] reported a unique case of coexistent HP and extrinsic asthma after repeated exposure to the dusty farm field, suggesting an aetiological relationship between these two distinct allergic pulmonary processes. However, most of the patients with HP caused by organic dust particles do not have concomitant airway inflammation. Several reports $[6,8-11]$ on combined HP and bronchial asthma after exposure to isocyanates suggest that co-existant HP and airway inflammation are induced by isocyanates rather than by organic dust particles. The present findings support this view.

Whether these histological changes were caused by the same mechanism as isocyanate-induced HP in humans remains unknown. However, the interstitial pneumonitis-like lesions described here developed only by the procedure of inhalation, and were caused at least a few hours after the last exposure only in sensitized animals. The changes resembled HP in humans caused by isocyanates [5, 7-11], as well as that caused by organic dust particles [16], including farmers' lung [22], bird fanciers' disease [23], and summer-type HP in Japan [24]. The pathological changes were most prominent in sensitized guinea-pigs killed $24 \mathrm{~h}$ after the last exposure (fig. 4).

In conclusion, this study shows that repeated exposure to TDI following sensitization by TDI inhalation caused interstitial pneumonitis-like lesions in guinea-pigs which resembled isocyanate-induced HP in humans. Whether isocyanate-induced HP in humans is caused exclusively by an immunological mechanism or not, and why there are some pathological differences between isocyanate-induced HP and HP induced by organic dust particles remain unknown. The repeated exposure of guinea-pigs to TDI by our method is helpful for developing interstitial pneumonitis-like lesions which mimic isocyanate-induced HP in humans. Consequently, analysis of the lesions developed in the present study may contribute to the studies on the pathophysiology and the pathogenetic mechanisms of isocyanate-induced hypersensitivity pneumonitis in more detail.

Acknowledgements: The authors thank K. Tanaka for his helpful comments, and T. Homma and K. Yasuoka for their technical assistance.

\section{References}

1. Musk AW, Peters JM, Wegman DH. Isocyanates and respiratory disease: current status. Am J Ind Med 1988; 13: 331-349.

2. Butcher BT, Jones RN, O'Neil CE, et al. Longitudinal study of workers employed in the manufacture of toluene diisocyanate. Am Rev Respir Dis 1977; 116: 411-421.

3. Mapp CE, Corona PC, Marzo ND, Fabbri LM. Persistent asthma due to isocyanates. Am Rev Respir Dis 1988; 137: 1326-1329.

4. Charles J, Bernstein A, Jones B, et al. Hypersensitivity pneumonitis after exposure to isocyanates. Thorax 1976; 31: 127-136.

5. Fink JN, Schlueter DP. Bathtub refinishers' lung: an unusual response to toluene diisocyanate. Am Rev Respir Dis 1978; 118: 955-959.

6. Zeiss CR, Kanellakes TM, Bellone JD, Levitz D, Pruzansky JJ, Patterson R. Immunoglobulin E-mediated asthma and hypersensitivity pneumonitis with precipitating antihapten antibodies due to diphenylmethane diisocyanate (MDI) exposure. J Allergy Clin Immunol 1980; 65: 346-352.

7. Malo JL, Zeiss CR. Occupational hypersensitivity pneumonitis after exposure to diphenylmethane diisocyanate. Am Rev Respir Dis 1982; 125: 113-116.

8. Malo JL, Ouimet G, Cartier A, Levitz D, Zeiss CR. Combined alveolitis and asthma due to hexamethylene diisocyanate (HDI), with demonstration of crossed respiratory and immunologic reactivities to diphenylmethane diisocyanate (MDI). J Allergy Clin Immunol 1983; 72: 413-419.

9. Baur X, Dewair M, Rommelt H. Acute airway obstruction followed by hypersensitivity pneumonitis in an isocyanate (MDI) worker. J Occup Med 1984; 26: 285-287.

10. Mapp CE, Vecchio LD, Boschetto P, Fabbri LM. Combined asthma and alveolitis due to diphenylmethane diisocyanate (MDI) with demonstration of no crossed respiratory reactivity to toluene diisocyanate (TDI). Ann Allergy 1985; 54: 424-429.

11. Bando T, Noda $\mathrm{Y}$, Hirose J, et al. A case of hypersensitivity pneumonitis induced by toluene diisocyanate presenting with transient bronchoconstriction. Jap J Thorac Dis 1993; 31: 1297-1302.

12. Fabbri LM, Danieli D, Crescioli S, et al. Fatal asthma in a subject sensitized to toluene diisocyanate. Am Rev Respir Dis 1988; 137: 1494-1498.

13. Patterson R, Nugent KM, Harris KE, Eberle ME. Immunologic hemorrhagic pneumonia caused by isocyanates. Am Rev Respir Dis 1990; 141: 226-230.

14. Tanaka K, Kawai M, Maekawa N. Experimental model of asthma by toluene diisocyanate (TDI). Bull Chest Dis Res Inst Kyoto Univ 1983; 16: 1-9.

15. Richerson HB, Suelzer MT, Swanson PA, Butler JE, Kopp WC, Rose EF. Chronic hypersensitivity pneumonitis produced in the rabbit by the adjuvant effect of inhaled muramyl dipeptide (MDP). Am J Pathol 1982; 106: 409-420.

16. Coleman A, Colby TV. Histological diagnosis of extrinsic allergic alveolitis. Am J Surg Pathol 1988; 12: 514-518.

17. Gordon T, Sheppard D, McDonald DM, Distefano S, Scypinski L. Airway hyperresponsiveness and inflammation induced by toluene diisocyanate in guinea-pigs. Am Rev Respir Dis 1985; 132: 1106-1112. 
18. Cibulas W, Murlas CG, Miller ML, et al. Toluene diisocyanate-induced airway hyperreactivity and pathology in the guinea-pig. J Allergy Clin Immunol 1986; 77: 828-834.

19. Saetta M, Stefano AD, Maestrelli P, et al. Airway mucosal inflammation in occupational asthma induced by toluene diisocyanate. Am Rev Respir Dis 1992; 145: 160-168.

20. Saetta M, Maestrelli P, Stefano AD, et al. Effect of cessation of exposure to toluene diisocyanate (TDI) on bronchial mucosa of subjects with TDI-induced asthma. Am Rev Respir Dis 1992; 145: 169-174.
21. Karr RM, Kohler PF, Salvaggio JE. Hypersensitivity pneumonitis and extrinsic asthma: an unusual association. Chest 1978; 74: 98-102.

22. Reyes CN, Wenzel FJ, Lawton BR, Emanuel DA. The pulmonary pathology of farmers' lung disease. Chest 1982; 81: 142-151.

23. Hendrick D, Faux J, Marshall R. Budgerigar-fanciers' lung. $B r$ Med $J$ 1978; 2: 81-84.

24. Ando M, Arima K, Yoneda R, Tamura M. Japanese summer-type hypersensitivity pneumonitis. Am Rev Respir Dis 1991; 144: 765-769. 